\title{
A molecular survey of febrile cases in malaria-endemic areas along China-Myanmar border in Yunnan province, People's Republic of China
}

\author{
Xia Zhou ${ }^{1,2, a}$, Ji-Lei Huang ${ }^{1,2, a}$, Metoh Theresia Njuabe ${ }^{2,3}$, Sheng-Guo Li $^{4}$, Jun-Hu Chen ${ }^{2, *}$, \\ and Xiao-Nong Zhou ${ }^{2, *}$ \\ 1 Department of Parasitology, Medical College of Soochow University, Suzhou 215123, China \\ 2 National Institute of Parasitic Diseases, Chinese Center for Disease Control and Prevention, WHO Collaborating Centre for Malaria, \\ Schistosomiasis and Filariasis, Key Laboratory of Parasite and Vector Biology Ministry of Health, Shanghai 200025, China \\ 3 Department of Biochemistry, Faculty of Sciences, University of Bamenda North-west Province, Cameroon \\ 4 Tengchong Center for Disease Control and Prevention, Tengchong 679100, Yunnan, China
}

Received 20 February 2014, Accepted 12 June 2014, Published online 23 June 2014

\begin{abstract}
Background: Imported malaria is a major threat to neighboring malaria-eliminating countries such as P.R. China and is difficult to monitor. A molecular survey of febrile patients with a history of traveling abroad along the Myanmar-China endemic border areas from January 2008 to August 2012 was carried out. The rates of infection with species of Plasmodium and compliance of microscopy diagnosis with nested PCR (Polymerase Chain Reaction) results were calculated. Results: Plasmodium genus-specific nested PCR confirmed that 384 cases were positive. Further species-specific nested PCR showed that the rate of Plasmodium vivax infection was 55\% (213/384); that of Plasmodium falciparum was $21 \%(81 / 384)$ and $17 \%(67 / 384)$ of cases were co-infection cases of $P$. vivax and $P$. falciparum; the remaining $6 \%(23 / 384)$ of cases were caused by other species, such as Plasmodium ovale, P. malaria, P. knowlesi or mixed infections of Plasmodium. In total there was 13\% (50/384) false microscopy diagnosis including 6\% $(22 / 384)$ error in species diagnosis and 7\% (28/384) undiagnosed cases in co-infection or low parasitemia malaria cases. Conclusions: This study indicates that there are considerable numbers of malaria cases in the China-Myanmar endemic border areas that remain undiagnosed or misdiagnosed by microscopy, especially in low-level and/or complex co-infection cases. It is urgent to develop accurate rapid diagnostic tests and apply PCR confirmation for efficient surveillance.
\end{abstract}

Key words: Malaria, Nested PCR, Diagnosis, Myanmar, P.R. China.

Résumé - Une étude moléculaire des cas de paludisme fébrile dans les régions endémiques le long de la frontière Chine-Myanmar dans la Province du Yunnan, République Populaire de Chine. Introduction : Le paludisme importé est une menace majeure pour les pays voisins qui éliminent le paludisme tels que République Populaire de Chine et est difficile à surveiller. Une enquête moléculaire a été réalisée sur des patients fébriles avec une histoire de voyage à l'étranger dans les zones endémiques frontalières Myanmar - Chine de janvier 2008 à août 2012. Les taux d'infection des espèces de Plasmodium et de conformité du diagnostic microscopique et des résultats PCR nichée ont été calculés. Résultats : la PCR nichée du genre Plasmodium a confirmé que 384 cas étaient positifs au genre. La PCR nichée spécifique aux espèces a montré que le taux d'infection à $P$. vivax était de $55 \%(213 / 384)$, celui de $P$. falciparum de $21 \%(81 / 384)$ et que $17 \%$ (67/384) des cas étaient des cas de coinfection de $P$. vivax et $P$. falciparum. Les $6 \%$ restants (23/384) des cas étaient causés par des infections d'autres espèces, telles que P. ovale, P. malariae, P. knowlesi ou des infections de Plasmodium mélangées. Au total il y avait $13 \%(50 / 384)$ de faux diagnostics microscopiques, y compris $6 \%$ (22/384) d'erreurs de diagnostic d'espèce et $7 \%(28 / 384)$ de diagnostics manquant dans les cas de coinfection ou les cas à parasitémie faible. Conclusions : L'étude indique que des nombres considérables de cas de paludisme dans les zones frontalières endémiques entre Chine et Myanmar restent non diagnostiqués ou mal diagnostiqués par la microscopie, surtout dans le cas de parasitémie faible ou de coinfections complexes. Il est urgent de développer des tests de diagnostic rapide et précis et d'appliquer une confirmation par PCR pour une surveillance efficace.

\footnotetext{
${ }^{\text {a }}$ These authors with equal contributions to this paper.

*Corresponding authors: junhuchen@hotmail.com; ipdzhouxn@shl63.net
} 


\section{Introduction}

Malaria is a major infectious disease in the Greater Mekong Subregion (GMS) countries, namely P.R. China, Laos, Myanmar, and Vietnam. In the GMS, Myanmar accounts for approximately one-fifth of the region's population, more than half of the malaria cases and approximately three-quarters of malaria deaths. "Border malaria" is extremely difficult to monitor; frequent malaria introductions by migratory human populations constitute a major threat to neighboring, malariaeliminating countries [9]. Yunnan province still has the highest transmission area in P.R. China, particularly in the southern border areas adjacent to Myanmar [7, 8, 30]. In the malaria elimination program, routine surveillance uses the standard method for detection of Plasmodium spp. infection by examining Giemsa-stained blood smears under the microscope. Although effective and inexpensive, the method is laborious and timeconsuming and its sensitivity drops with the decrease in parasitemia during the disease elimination stage [2]. The Polymerase Chain Reaction (PCR) method has been widely used for the detection and identification of malaria parasites. It was also successfully used to detect parasites in mixed infections, as well as those with low parasitemia $[4,26]$. A variety of PCR-based assays have been described for the specific diagnosis of all the five species of Plasmodium, i.e. Plasmodium falciparum, P. vivax, P. ovale, P. malariae, and P. knowlesi [23, 25, 32, $22,5]$, one of which is based on the sequence of the small subunit ribosomal RNA (ssrRNA) gene used to detect human Plasmodium spp. [24].

A molecular survey was carried out of samples collected randomly in the malaria-endemic area along the border line of southern China, in Yunnan province from January 2008 to August 2012. The present study aims to evaluate PCR amplification in comparison with light microscopy examination.

\section{Materials and methods}

\section{Ethical clearance}

The research proposal was approved by the ethics committee at the National Institute of Parasitic Diseases, Chinese Center for Disease Control and Prevention, in particular regarding collecting blood samples from local residents or migrant workers by finger prick. Written informed consent was obtained from all adult participants and from the parents, or legal guardians of children. Any participant found to be parasitologically positive received anti-malaria treatment, except for those with contraindications.

\section{Patient samples and microscopy examination}

The population of the Tengchong village is served by a general hospital, a polyclinic at the village's Center for Disease Control and Prevention (CDC), and 15 government health clinics. A total of 562 blood specimens were collected from febrile patients in China-Myanmar endemic border areas at Tengchong Hospital, the polyclinic of the village's CDC and the government health clinics. All these patients in Tengchong village come from different countries and most of (82.6\%, 464/562) these febrile patients had a history of being abroad in Myanmar during the last year. Finger-prick blood samples were collected and the Giemsa-stained blood smears were examined by microscope from January 2008 to August 2012. Thick blood smears were examined under $1000 \times$ magnification by microscopists with extensive experience in identification of malaria parasites. The thick film was considered positive when malaria parasites were present and negative if no parasites were seen after 500 leukocytes were counted. In total, 373 samples including 214 $P$. vivax infection cases, 96 P. falciparum cases and 63 cases of vivax and falciparum co-infection were referred to as the Microscopy-Positive group (MP group). The remaining 189 samples were the Microscopy-Negative group (MN group). Parasite density per 200 leukocytes was counted, then calculated as the number of parasites per microliter by assuming a leukocyte count of 7000 .

\section{DNA extraction}

DNA was extracted from the blood spots using the QIAamp DNA Mini Kit (QIAGEN China (Shanghai) Co., Ltd.) according to the manufacturer's instructions and the method of Foley et al. [12]. Approximately $30 \mu \mathrm{L}$ of blood were used per filter spot. The final template volume obtained after Chelex extraction was $150 \mu \mathrm{L}$. Throughout the process, negative controls were included to ensure lack of contamination.

\section{PCR amplification}

Nested PCR amplification was performed according to Snounou et al. [24] using a Biometra Thermocycler (England). For the first PCR, $1 \mu \mathrm{L}$ of template DNA was added to a $20-\mu \mathrm{L}$ PCR mixture consisting of $0.4 \mathrm{M}$ of each primer (rPLU1 and rPLU5), $10 \mu \mathrm{L} 2 \times$ Taq PCR MasterMix (Tiangen Biotech, Beijing, China) containing $0.1 \mathrm{U}$ Taq polymerase $/ \mu \mathrm{L}, 500 \mu \mathrm{M}$ deoxynucleotide triphosphates (dNTP), $3 \mathrm{mM} \quad \mathrm{MgCl}_{2}$, $100 \mathrm{mM} \mathrm{KCl}, 20 \mathrm{mM}$ Tris- $\mathrm{HCl}, \mathrm{pH}$ 8.3. DNA amplification was carried out under the following conditions: $94{ }^{\circ} \mathrm{C}$ for $5 \mathrm{~min}, 30$ cycles of $\left(94{ }^{\circ} \mathrm{C}\right.$ for $30 \mathrm{~s}, 55^{\circ} \mathrm{C}$ for $30 \mathrm{~s}$, and $72{ }^{\circ} \mathrm{C}$ for $1 \mathrm{~min}$ ), followed by a final extension at $72{ }^{\circ} \mathrm{C}$ for 5 min. One microliter of the first PCR product was used in the second amplification. Conditions and concentrations used for the second amplification were identical to those used for the first, except that rPLU3/rPLU4 were used as primers (Table 1) and amplification was performed over 35 cycles. The size of the DNA target, amplified by these outer primers, is about $1600-1700 \mathrm{bp}$ and by the inner primers is $235 \mathrm{bp}$. These primers are genus-specific, and can therefore amplify the target sequences from all five species of the human malaria parasite, P. falciparum, P. vivax, P. ovale, P. malariae and P. knowlesi. Detection of Plasmodium species was performed using five species-specific primer pairs. Because of the primer pair Pmk8 and Pmkr9 may cross-hybridize with the corresponding sequence of $P$. vivax because of the high similarity of $18 \mathrm{~S}$ SSU rRNA gene sequences among these parasites [13]. Knowlesi malaria infection cases were confirmed by a new Iset of primers (Pkr140-5F, Pkr140-5R) with application in a 
Table 1. Primer sequences for PCR detection of five human malaria parasites

\begin{tabular}{|c|c|c|c|c|}
\hline & Plasmodium spp & Size (bp) & Primers & Primer sequences $\left(5^{\prime} \rightarrow 3^{\prime}\right)$ \\
\hline \multirow[t]{2}{*}{ Nested 1st round primers } & Genus-specific & $1600-1700$ & rPLU1 & TCAAAGATTAAGCCATGCAAGTGA \\
\hline & & & rPLU5 & CCTGTTGTTGCCTTAAACTTC \\
\hline \multirow[t]{2}{*}{ Nested 2nd round primers } & Genus-specific & 235 & rPLU3 & TTTTTATAAGGATAACTACGGAAAAGCTGT \\
\hline & & & rPLU4 & TACCCGTCATAGCCATGTTAGGCCAATACC \\
\hline \multirow[t]{10}{*}{ Nested 2nd round species-specific primers } & P. vivax & 121 & rVIV1 & CGCTTCTAGCTTAATCCACATAACTGATAC \\
\hline & & & rVIV2 & ACTTCCAAGCCGAAGCAAAGAAAGTCCTTA \\
\hline & P. falciparum & 206 & rFAL1 & TTAAACTGGTTTGGGAAAACCAAATATATT \\
\hline & & & rFAL2 & ACACAATGAACTCAATCATGACTACCCGTC \\
\hline & P. ovale & 226 & rOVA1 & ATCTCTTTTGCTATTTTTTAGTATTGGAGA \\
\hline & & & rPLU2 & ATCTAAGAATTTCACCTCTGACATCTG \\
\hline & P. malariae & 145 & rMAL1 & ATAACATAGTTGTACGTTAAGAATAACCCC \\
\hline & & & rMAL2 & AAAATTCCCATGCATAAAAATTATACAAA \\
\hline & P. knowlesi & 153 & Pmk8 & GTTAGCGAGAGCCACAAAAAAGCGAAT \\
\hline & & & Pmkr9 & ACTCAAAGTAACAAAATCTTCCGTA \\
\hline \multirow[t]{2}{*}{ Single-Step PCR primers } & P. knowlesi & 200 & Pkr140-5F & CAGAGATCCGTTCTCATGATTTCCATGG \\
\hline & & & Pkr140-5R & CTRAACACCTCATGTCGTGGTAG \\
\hline
\end{tabular}

Single-Step PCR Assay for the detection of P. knowlesi [18]; all the primers were also applied: see Table 1 . If the genus-specific nested PCR results were positive, these first PCR products were used as the template in the next species nested PCR amplification under the same conditions. The negative control reaction was established in each amplification reaction. The positive controls of $P$. falciparum and $P$. vivax were, respectively, set by the 3D7 strain of $P$. falciparum and isolate of $P$. vivax from a malaria patient of Yunnan province preserved by our laboratory. Electrophoresis of the PCR products was performed using $2 \%$ agarose gel followed by Gold-View staining and visualizing under a UV light. All the positive results of infected $P$. ovale, $P$. knowlesi and $P$. malariae infection cases and several $P$. vivax and $P$. falciparum ones plus all the co-infection cases were again confirmed by sequencing of PCR products by BGI Company, China. Sequences obtained by direct sequencing of nested-PCR products of second rounds from these co-infection cases above were aligned by using the ClustalW method (EMBL-EBI, Hixton, and Cambridge, UK) and analyzed by MEGA version 5.1 (http://mega.software.informer.com/5.1b/)

\section{Results and discussions}

All 562 specimens were collected and the Giemsa-stained blood smears were examined under the microscope. In total, 373 cases were positive by microscopy (MP group), while the remaining 189 samples were diagnosed as negative (MN group). Plasmodium genus-specific nested PCR confirmed that 384 cases were positive (371 cases from the MP group with 214 cases of $P$. vivax infections, 96 cases of $P$. falciparum infections and 61 cases of co-infections of $P$. vivax and $P$. falciparum, and 13 from the MN group of 189 febrile patients). So, the nested PCR confirmed all of the MP group malaria cases (100\%, 371/ $371)$ and diagnosed 13 malaria cases in the $\mathrm{MN}$ group $(6.9 \%$, $13 / 189$ ) which may have been ignored because of the low parasitemia conditions. Further species-specific nested PCR identified that among the 384 malaria cases, the $P$. vivax infection rate was $55.5 \%(213 / 384)$, that of $P$. falciparum was $21.1 \%(81 / 384)$ and $17.4 \%(67 / 384)$ of cases were co-infections of $P$. vivax and $P$. falciparum, with $6.0 \%(23 / 384)$ of cases caused by other infections, including five cases of $P$. ovale and three of $P$. malariae; and various cases of mixed infections: two cases of P. knowlesi and P. falciparum, three cases of P. vivax, P. falciparum and $P$. ovale, two cases of $P$. vivax and $P$. ovale, two cases of $P$. falciparum and $P$. ovale, two cases of $P$. falciparum and $P$. malariae, one case of $P$. vivax, $P$. falciparum and $P$. ovale, one case of $P$. vivax, $P$. malariae and $P$. ovale, one case of $P$. vivax, $P$. falciparum, $P$. malariae and $P$. ovale, and one case of $P$. malariae and $P$. ovale. In total there was $13.0 \%$ (50/384) error in microscopy diagnosis including 5.7\% (22/ $384)$ error in species diagnosis and 7.3\% (28/384) undiagnosed cases in co-infection or low parasitemia malaria cases (see the details of all the species of 384 malaria infection cases in Table 2 and Fig. 1). Phylogenetic trees were produced by using the neighbor-joining method in MEGA version 5.1 The case samples (denoted as Pv1, Pv2, Pm1, Pm2, Pk1, Pk2, Pf1, Pf2, Pf3, Po1, and Po2) clustered with other Plasmodium isolates (see Fig. 2).

The long border of $4061 \mathrm{~km}$ in southern Yunnan, directly adjacent to the northwest of Laos, northeast of Myanmar and north of Vietnam, is considered the most prevalent area of malaria in P.R. China [8]. Despite the reduction of malaria over the last decade in China, care must be taken to prevent any new foci of epidemics, after launching the National Malaria Eradication Program (NMEP) as a consequence of different factors. Control and eventual elimination of human parasitic diseases in P.R. China requires novel, innovative approaches, particularly in areas of diagnostics, mathematical modeling, monitoring, evaluation, surveillance and public health response [6]. In addition, the movement of sections of the population that includes tourism, border trade business, traveling and holidays, particularly in those free ports on the border, may affect the mode of severity and endemics of malaria in this region [8].

Low-level infection appears to be common across malariaendemic areas, often as complex mixed infections. A number of studies demonstrated that PCR is more reliable than microscopy in detection of malaria in low parasitemic areas [14, 20, 21]. 
Table 2. Plasmodium species identified in 384 malaria patients in China-Myanmar endemic border areas in Yunnan province, January 2008 to August 2012

\begin{tabular}{|c|c|c|c|c|c|}
\hline \multirow[t]{3}{*}{ Species-specific PCR results } & \multicolumn{3}{|c|}{ Microscopy positive (371) } & \multirow{3}{*}{$\begin{array}{c}\text { Microscopy negative } \\
189\end{array}$} & \multirow[t]{3}{*}{ Total no. $(\%)$} \\
\hline & P. vivax & P. falciparum & P. vivax $/ P$. falciparum & & \\
\hline & 214 & 96 & 61 & & \\
\hline P. vivax & 195 & 6 & 0 & 12 & $213(55.5)$ \\
\hline P. falciparum & 6 & 74 & 0 & 1 & $81(21.1)$ \\
\hline P. vivax $/$ P. falciparum & 3 & 5 & 59 & 0 & $67(17.4)$ \\
\hline P. ovale & 2 & 2 & 1 & 0 & $5(1.3)$ \\
\hline P. malariae & 2 & 0 & 1 & 0 & $3(0.8)$ \\
\hline P. falciparum/P. vivax/P. ovale & 1 & 2 & 0 & 0 & $3(0.8)$ \\
\hline P. vivax/P. ovale & 2 & 0 & 0 & 0 & $2(0.5)$ \\
\hline P. falciparum/P. knowlesi & 0 & 2 & 0 & 0 & $2(0.5)$ \\
\hline P. falciparum/P. malariae & 0 & 2 & 0 & 0 & $2(0.5)$ \\
\hline P. falciparum/P. ovale & 1 & 1 & 0 & 0 & $2(0.5)$ \\
\hline P. falciparum/P. vivax/P. malariae & 1 & 0 & 0 & 0 & $1(0.3)$ \\
\hline P. ovale/P. malariae & 1 & 0 & 0 & 0 & $1(0.3)$ \\
\hline P. vivax/P. ovale/P. malariae & 1 & 0 & 0 & 0 & $1(0.3)$ \\
\hline P. falciparum/P. vivax/P. ovale/P. malariae & 0 & 1 & 0 & 0 & $1(0.3)$ \\
\hline
\end{tabular}

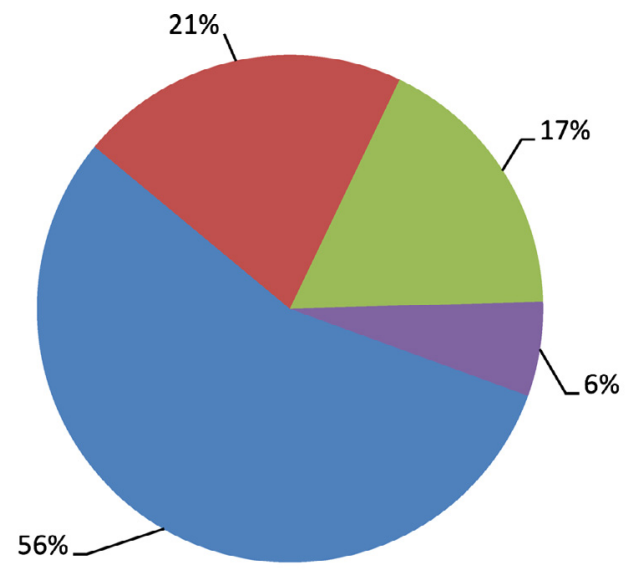

$\square$. vivax $\backsim P$. falciparum $\backsim P$. vivax $/ P$. falciparum $₫$ other $P$. $s p p$

Figure 1. Plasmodium species identified in 384 malaria patients from China-Myanmar endemic areas form Yunnan province, January 2008 to August 2012.

Several malaria infections from endemic countries are reported to be sub-patent, with very low parasitemia [28]. Due to false negative diagnosis by microscopy in endemic areas, a number of patients remain untreated and they may play a role as carriers of malaria parasites. Microscopy analysis is currently used for identification of Plasmodium species, a technique appropriate for routine clinical diagnosis. However, its sensitivity is limited and diagnostic accuracy depends greatly on the particular expertise of laboratory staff. The detection limit of malaria parasites by microscopy is $20-30$ parasites $/ \mu \mathrm{L}$ of blood [17]. Frequently, however, due to specimen load pressure, inadequate staining and poorly maintained microscopes, even this limit may be decreased in both quality and quantity. The PCR method not only detected almost all of the microscopy-positive samples, but also detected mixed infections and malaria cases that were not diagnosed by microscope $[4,14]$.

It is reported that $P$. ovale, rarely seen in P.R. China, was distributed widely in Myanmar [29]; here, we detected more than $3 \%$ ovale malaria cases in China-Myanmar endemic border areas. Our species detection work also improved as Kawamoto et al. [16] reported that there might be a higher prevalence of $P$. ovale, and P. malariae in East Asia than previously reported when using alternative diagnostic methods, such as acridine orange staining and PCR-based methods. The rate of P. knowlesi co-infections with other malaria parasites was reported to be about 30\% in southern Myanmar near Yunnan Province of China [15]. The P. knowlesi co-infection rate detected in this study was much lower than that reported in southern Myanmar, which may be because all the field samples we collected were mainly in the villages adjacent to northeastern Myanmar, so only two knowlesi malaria cases were detected. To establish long-term cooperation in prevention and control of important infectious diseases in cross-border areas, a proposal for malaria prevention and control in border areas of Yunnan has been suggested. A more sensitive and specific diagnosis method is needed to obtain a rapid-response emergency mechanism for the important infectious diseases [1].

Artemisinin resistance in $P$. falciparum malaria has already been reported [10, 19]. Uhlemann and Fidock [27] reported that Plasmodium loss of malarial susceptibility to artemisinin in Thailand suggested that the resistance gene also exists in $P$. vivax-endemic areas. The latest research work in northern Angola has demonstrated that because $P$. malariae and $P$. ovale are sympatric with $P$. falciparum across the continent and are frequently present as co-infections, populations of $P$. falciparum previously dominated by chloroquine (CQ)-resistant genotypes are now under different drug selection pressures. In short, it is essential that the treatment of malaria patients depends on the correct diagnosis of malaria species, particularly with the spread of parasitic resistance to antimalarial drugs $[3,11]$. Thus, controlling malaria may face long-term difficulties.

Our latest study showed that human babesiosis caused by another intraerythrocytic protozoan, Babesia microti, is emerging in China-Myanmar border areas in Yunnan province, P.R. China [31]. So, the co-prevalence of Babesia in malariaendemic regions is possible and sometimes babesiosis has been 


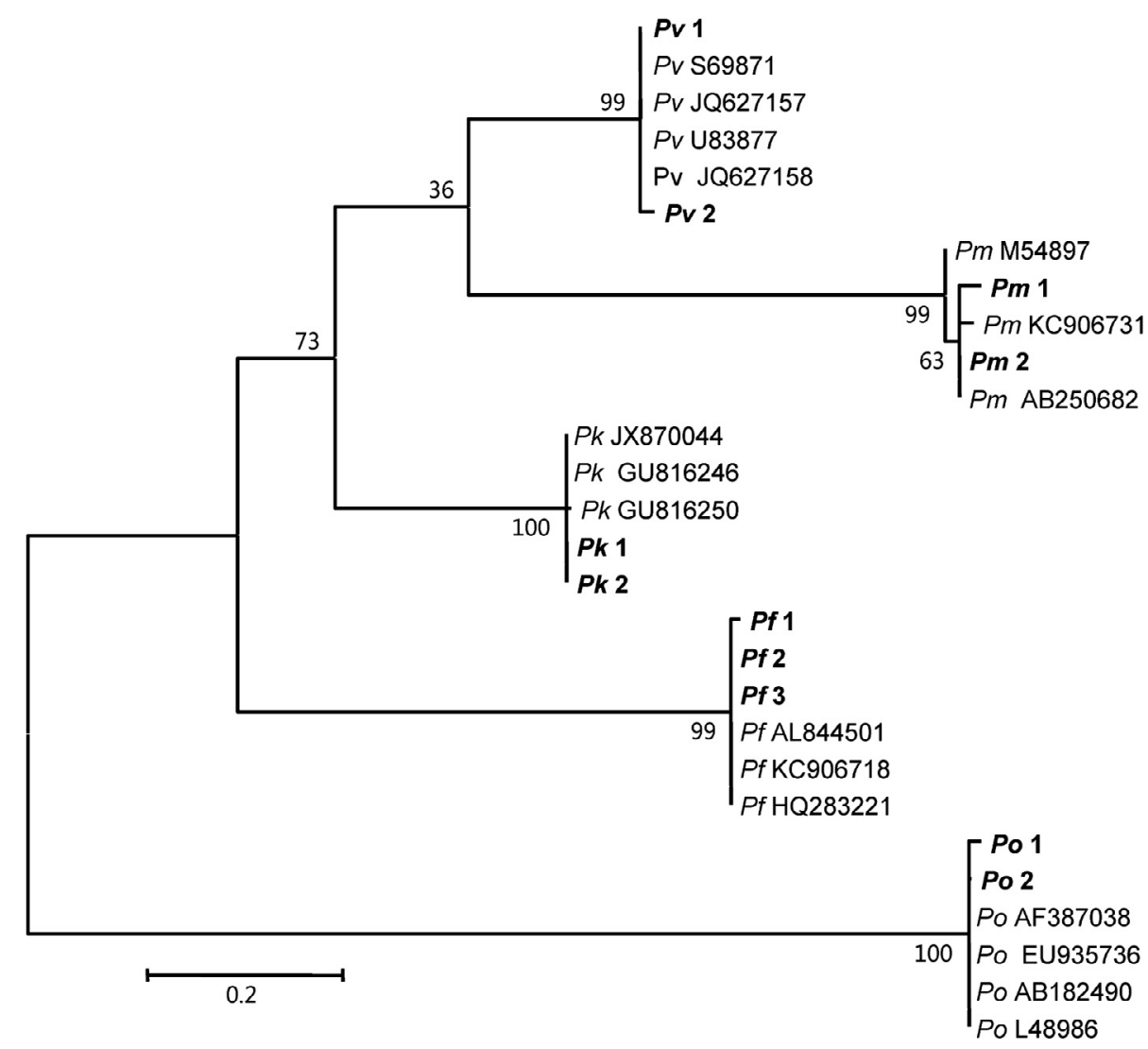

Figure 2. Phylogenetic trees based on the partial sequences of $18 \mathrm{~S}$ ribosomal RNA gene of five Plasmodium from malaria patients in ChinaMyanmar endemic areas from Yunnan province, January 2008 to August 2012. Phylogenetic analysis produced by the neighbor-joining method using MEGA version 5.1 software. Partial sequences of 18S ribosomal RNA of our case study samples are denoted as Pv1, Pv2, Pm1, Pm2, Pk1, Pk2, Pf1, Pf2,Pf3, Po1, and Po2 in bold face with other Plasmodium species. Scale bar indicates nucleotide substitutions per site.

diagnosed as malaria due to the similarities of symptoms and morphology. Laboratories in babesiosis-endemic areas need broad access to modern diagnostic methods for a more rapid and reliable microbiological diagnosis in cases of suspected cases of malaria and/or human babesiosis. We should also pay attention to $P$. ovale, $P$. knowlesi and $P$. malariae as well as Babesia, for there may be co-infection with the other two dominant Plasmodium species.

\section{Conclusion}

This study indicates that there are considerable numbers of malaria cases in the China-Myanmar endemic border areas that remain undiagnosed or misdiagnosed by microscopy, especially in low-level and/or complex co-infection cases. It is urgent to develop accurate, rapid diagnostic tests suitable for these intraerythrocytic protozoans besides the two dominant plasmodium species, and apply PCR confirmation for accurate surveillance.

Acknowledgements. XZ and JLH conceived the study, collected the data and analyzed it, and drafted the manuscript. MTN and SGL revised the manuscript and provided interpretation of the findings. JHC conceived the project and provided technical support for data collection and analysis. XNZ conceived the study and revised the manuscript. All authors read and approved the final manuscript.

This work was financially supported by the Special Fund for Health Research in the Public Interest (Grant No. 201202019) and was supported in part by the Foundation of National Science and Technology Major Program (Grant No. 2012ZX10004220). All authors declared no conflict of interest.

\section{References}

1. Anonymous. 2012. Inaugural meeting of the malaria policy advisory committee to the WHO: conclusions and recommendations. Malaria Journal, 11, 137.

2. Agabani HM, el Hag IA, el Toum IA, Satti M, el Hassan AM. 1994. Fluorescence microscopy using a light microscope fitted with an interference filter for the diagnosis of malaria. Transactions of the Royal Society of Tropical Medicine and Hygiene, 88(1), 61.

3. Aguiar AC, Santos Rde M, Figueiredo FJ, Cortopassi WA, Pimentel AS, Franca TC, Meneghetti MR, Krettli AU. 2012. Antimalarial activity and mechanisms of action of two novel 4-aminoquinolines against chloroquine-resistant parasites. PLoS One, 7(5), e37259. 
4. Carrasquilla G, Banguero M, Sanchez P, Carvajal F, Barker RH Jr, Gervais GW, Algarin E, Serrano AE. 2000. Epidemiologic tools for malaria surveillance in an urban setting of low endemicity along the Colombian Pacific coast. American Journal of Tropical Medicine and Hygiene, 62(1), 132-137.

5. Chen JH, Lu F, Lim CS, Kim JY, Ahn HJ, Suh IB, Takeo S, Tsuboi T, Sattabongkot J, Han ET. 2010. Detection of Plasmodium vivax infection in the Republic of Korea by loop-mediated isothermal amplification (LAMP). Acta Tropica, 113(1), 61-65.

6. Chen JH, Wang H, Chen JX, Bergquist R, Tanner M, Utzinger J, Zhou XN. 2012. Frontiers of parasitology research in the People's Republic of China: infection, diagnosis, protection and surveillance. Parasites \& Vectors, 5, 221.

7. Cui L, Yan G, Sattabongkot J, Cao Y, Chen B, Chen X, Fan Q, Fang Q, Jongwutiwes S, Parker D, Sirichaisinthop J, Kyaw MP, Su XZ, Yang H, Yang Z, Wang B, Xu J, Zheng B, Zhong D, Zhou G. 2012. Malaria in the Greater Mekong Subregion: heterogeneity and complexity. Acta Tropica, 121(3), 227-239.

8. Cui L, Yan G, Sattabongkot J, Chen B, Cao Y, Fan Q, Parker D, Sirichaisinthop J, Su XZ, Yang H, Yang Z, Wang B, Zhou G. 2012. Challenges and prospects for malaria elimination in the Greater Mekong Subregion. Acta Tropica, 121(3), 240-245.

9. Delacollette C, D'Souza C, Christophel E, Thimasarn K, Abdur R, Bell D, Dai TC, Gopinath D, Lu S, Mendoza R, Ortega L, Rastogi R, Tantinimitkul C, Ehrenberg J. 2009. Malaria trends and challenges in the Greater Mekong Subregion. Southeast Asian Journal of Tropical Medicine and Public Health, 40(4), 674-691.

10. Dondorp AM, Nosten F, Yi P, Das D, Phyo AP, Tarning J, Lwin KM, Ariey F, Hanpithakpong W, Lee SJ, Ringwald P, Silamut K, Imwong M, Chotivanich K, Lim P, Herdman T, An SS, Yeung S, Singhasivanon P, Day NP, Lindegardh N, Socheat D, White NJ. 2009. Artemisinin resistance in Plasmodium falciparum malaria. New England Journal of Medicine, 361(5), 455-467.

11. Fancony C, Gamboa D, Sebastiao Y, Hallett R, Sutherland C, Sousa-Figueiredo JC, Nery SV. 2012. Various pfert and pfmdr1 Genotypes of Plasmodium falciparum Cocirculate with P. malariae, P. ovale spp., and P. vivax in Northern Angola. Antimicrobial Agents and Chemotherapy, 56(10), 5271-5277.

12. Foley M, Ranford-Cartwright LC, Babiker HA. 1992. Rapid and simple method for isolating malaria DNA from fingerprick samples of blood. Molecular and Biochemical Parasitology, 53(1-2), 241-244.

13. Imwong M, Tanomsing N, Pukrittayakamee S, Day NP, White NJ, Snounou G. 2009. Spurious amplification of a Plasmodium vivax small-subunit RNA gene by use of primers currently used to detect P. knowlesi. Journal of Clinical Microbiology, 47(12), 4173-4175.

14. Jelinek T, Proll S, Hess F, Kabagambe G, von Sonnenburg F, Loscher T, Kilian AH. 1996. Geographic differences in the sensitivity of a polymerase chain reaction for the detection of Plasmodium falciparum infection. American Journal of Tropical Medicine and Hygiene, 55(6), 647-651.

15. Jiang N, Chang Q, Sun X, Lu H, Yin J, Zhang Z, Wahlgren M, Chen Q. 2010. Co-infections with Plasmodium knowlesi and other malaria parasites. Myanmar. Emerging Infectious Diseases, 16(9), 1476-1478.

16. Kawamoto F, Liu Q, Ferreira MU, Tantular IS. 1999. How prevalent are Plasmodium ovale and P. malariae in East Asia? Parasitology Today, 15(10), 422-426.
17. Kimura M, Miyak H, Kim HS, Tanabe M, Arai M, Kawai S, Yamane A, Wataya Y. 1995. Species-specific PCR detection of malaria parasites by microtiter plate hybridization: clinical study with malaria patients. Journal of Clinical Microbiology, 33(9), 2342-2346.

18. Lucchi NW, Poorak M, Oberstaller J, DeBarry J, Srinivasamoorthy G, Goldman I, Xayavong M, da Silva AJ, Peterson DS, Barnwell JW, Kissinger J, Udhayakumar V. 2012. A new single-step PCR assay for the detection of the zoonotic malaria parasite Plasmodium knowlesi. PLoS One, 7(2), e31848.

19. Noedl H, Se Y, Schaecher K, Smith BL, Socheat D, Fukuda MM. 2008. Evidence of artemisinin-resistant malaria in western Cambodia. New England Journal of Medicine, 359(24), 2619-2620.

20. Roper C, Elhassan IM, Hviid L, Giha H, Richardson W, Babiker H, Satti GM, Theander TG, Arnot DE. 1996. Detection of very low level Plasmodium falciparum infections using the nested polymerase chain reaction and a reassessment of the epidemiology of unstable malaria in Sudan. American Journal of Tropical Medicine and Hygiene, 54(4), 325-331.

21. Singh B, Cox-Singh J, Miller AO, Abdullah MS, Snounou G, Rahman HA. 1996. Detection of malaria in Malaysia by nested polymerase chain reaction amplification of dried blood spots on filter papers. Transactions of the Royal Society of Tropical Medicine and Hygiene, 90(5), 519-521.

22. Singh B, Kim Sung L, Matusop A, Radhakrishnan A, Shamsul SS, Cox-Singh J, Thomas A, Conway DJ. 2004. A large focus of naturally acquired Plasmodium knowlesi infections in human beings. Lancet, 363(9414), 1017-1024.

23. Snounou GL, Pinheiro L, Goncalves A, Fonseca L, Dias F, Brown KN, do Rosario VE. 1993. The importance of sensitive detection of malaria parasites in the human and insect hosts in epidemiological studies, as shown by the analysis of field samples from Guinea Bissau. Transactions of the Royal Society of Tropical Medicine and Hygiene, 87(6), 649-653.

24. Snounou G, Singh B. 2002. Nested PCR analysis of Plasmodium parasites. Methods in Molecular Medicine, 72, 189-203.

25. Snounou GS, Viriyakosol S, Jarra W, Thaithong S, Brown KN. 1993. Identification of the four human malaria parasite species in field samples by the polymerase chain reaction and detection of a high prevalence of mixed infections. Molecular and Biochemical Parasitology, 58(2), 283-292.

26. Snounou GS, Viriyakosol S, Zhu XP, Jarra W, Pinheiro L, do Rosario VE, Thaithong S, Brown KN. 1993. High sensitivity of detection of human malaria parasites by the use of nested polymerase chain reaction. Molecular and Biochemical Parasitology, 61(2), 315-320.

27. Uhlemann AC, Fidock DA. 2012. Loss of malarial susceptibility to artemisinin in Thailand. Lancet, 379(9830), 1928-1930.

28. Wen L, Li C, Lin M, Yuan Z, Huo D, Li S, Wang Y, Chu C, Jia R, Song H. 2011. Spatio-temporal analysis of malaria incidence at the village level in a malaria-endemic area in Hainan. China. Malaria Journal, 10, 88.

29. Win TT, Lin K, Mizuno S, Zhou M, Liu Q, Ferreira MU, Tantular IS, Kojima S, Ishii A, Kawamoto F. 2002. Wide distribution of Plasmodium ovale in Myanmar. Tropical Medicine \& International Health, 7(3), 231-239.

30. Zhou SS, Wang Y, Xia ZG. 2011. Malaria situation in the People's Republic of China in 2009. Zhongguo Ji Sheng Chong Xue Yu Ji Sheng Chong Bing Za Zhi, 29(1), 1-3. 
31. Zhou X, Li SG, Chen SB, Wang JZ, Xu B, Zhou HJ, Ge HX, Chen JH, Hu W. 2013. Co-infections with Babesia microti and Plasmodium parasites along the China-Myanmar border. Infectious Diseases of Poverty, 2(1), 24.
32. Zhu XP, Zhang XM, Zhou L, Yang YP, Gao X. 2002. Sequence analysis and genotypes of glutamate rich protein of Plasmodium falciparum isolates from different malaria endemic areas in China. Biomedical and Environmental Sciences, 15(1), 1-7.

Cite this article as: Zhou X, Huang J-L, Njuabe MT, Li S-G, Chen J-H \& Zhou X-N: A molecular survey of febrile cases in malariaendemic areas along China-Myanmar border in Yunnan province, People's Republic of China. Parasite, $2014,21,27$.

\section{OP PARASTE}

An international open-access, peer-reviewed, online journal publishing high quality papers on all aspects of human and animal parasitology

Reviews, articles and short notes may be submitted. Fields include, but are not limited to: general, medical and veterinary parasitology; morphology, including ultrastructure; parasite systematics, including entomology, acarology, helminthology and protistology, and molecular analyses; molecular biology and biochemistry; immunology of parasitic diseases; host-parasite relationships; ecology and life history of parasites; epidemiology; therapeutics; new diagnostic tools.

All papers in Parasite are published in English. Manuscripts should have a broad interest and must not have been published or submitted elsewhere. No limit is imposed on the length of manuscripts.

Parasite (open-access) continues Parasite (print and online editions, 1994-2012) and Annales de Parasitologie Humaine et Comparée (1923-1993) and is the official journal of the Société Française de Parasitologie. 